Ferreira, TA, Rodrigues, RC, Freitas, RF, Dias, ACP \& Lessa, AC. (2020). Whole foods and reuse of food: Knowing the practices Diamantina, Minas Gerais, Brasil. Research, Society and Development. 9(7): 1-19. e201973757.

\title{
Uso integral e reaproveitamento de alimentos: conhecendo as práticas de Diamantina,
} Minas Gerais, Brasil

Whole foods and reuse of food: Knowing the practices Diamantina, Minas Gerais, Brasil Uso integral y reutilización de alimentos: conociendo las prácticas de Diamantina, Minas

\section{Gerais, Brasil}

Recebido: 18/04/2020 | Revisado: 28/04/2020 | Aceito: 02/05/2020 | Publicado: 08/05/2020

Tássia Andrade Ferreira

ORCID: https://orcid.org/0000-0003-1463-4787

Universidade Federal dos Vales do Jequitinhonha e Mucuri, Brasil.

E-mail: tassiaandrade1@gmail.com

Renata Cristina Rodrigues

ORCID: https://orcid.org/0000-0002-6459-8553

Universidade Federal dos Vales do Jequitinhonha e Mucuri, Brasil.

E-mail: renata_ufvjm@yahoo.com.br

Ronilson Ferreira Freitas

ORCID: https://orcid.org/0000-0001-9592-1774

Universidade Estadual de Montes Claros, Brasil.

E-mail: ronnypharmacia@gmail.com

Ana Catarina Perez Dias

ORCID: https://orcid.org/0000-0002-6690-4139

Universidade Federal dos Vales do Jequitinhonha e Mucuri, Brasil.

E-mail: acatarina@hotmail.com

Angelina do Carmo Lessa

ORCID: http://orcid.org/0000-0003-0708-4799

Universidade Federal dos Vales do Jequitinhonha e Mucuri, Brasil.

E-mail: angelinalessa@hotmail.com

\section{Resumo}

Objetivou-se conhecer as práticas de aproveitamento integral, o desperdício e o destino dado às sobras alimentares pela população residente de Diamantina/MG. Participaram do estudo, 
200 moradores de 4 bairros da cidade atendidos por unidades de saúde da família. Foi aplicado um formulário semi-estruturado constando de dois blocos, o primeiro com características socioeconômicas e demográficas, e o segundo, relativo às práticas de preparo, conservação e consumo de alimentos. A perda de alimentos em casa antes do preparo/consumo foi observada em $41,4 \%$ dos participantes. Sobre o destino das sobras de alimentos $71,2 \%$ dos participantes disseram fazer o reaproveitamento das sobras. $\mathrm{O}$ aproveitamento integral dos alimentos foi observado em $47,2 \%$ dos participantes. Dessa forma conclui-se que o uso do aproveitamento integral de alimentos ainda é pouco praticado pela população, já a utilização das sobras é uma prática mais comum entre os entrevistados apesar do desperdício ainda ser grande em nossa sociedade.

Palavras-chave: aproveitamento integral, reaproveitamento de alimentos, desperdício.

\begin{abstract}
The objective was to know the practices of full use, waste and the destination given to food leftovers by the resident population of Diamantina/MG. The participants were residents of 4 neighbourhoods attended by family health units, with a number of 50 people per neighbourhood being defined, a semi-structured questionnaire consisting of two blocks was applied, the first with socioeconomic and demographic characteristics, and the second, relative to food preparation, conservation and consumption practices. The application was only carried out after the participant had received and signed the Free and Informed Consent Form (ICF) and was over 18 years old. The loss of food at home before preparation/consumption was observed in $41.4 \%$ of participants. Regarding the destination of leftover food, $71.2 \%$ of the participants said they had to reuse the leftovers. The full use of food was observed in $47.2 \%$ of the participants. Thus, it is concluded that the use of full use of food is still little practiced by the population, since the use of leftovers is a more common practice among respondents despite the fact that waste is still great among the population.
\end{abstract}

Keywords: full use, reuse of food, waste.

\title{
Resumen
}

El objetivo era conocer las prácticas de uso completo, desperdicio y el destino dado a los restos de comida por la población residente de Diamantina/MG. Los participantes eran residentes de 4 vecindarios atendidos por unidades de salud familiar, con un número definido de 50 personas por vecindario, se aplicó un cuestionario semiestructurado que consta de dos bloques, el primero con características socioeconómicas y demográficas, y el segundo, 
relativo a la preparación de alimentos, conservación y prácticas de consumo. La solicitud solo se llevó a cabo después de que el participante recibió y firmó el Formulario de consentimiento libre e informado (ICF) y tenía más de 18 años. La pérdida de alimentos en el hogar antes de la preparación/consumo se observó en el 41,4\% de los participantes. Con respecto al destino de los restos de comida, el 71,2\% de los participantes dijeron que tenían que reutilizar los restos. El uso completo de los alimentos se observó en el 47,2\% de los participantes. Por lo tanto, se concluye que el uso del uso completo de los alimentos todavía es poco practicado por la población, ya que el uso de las sobras es una práctica más común entre los encuestados a pesar del hecho de que el desperdicio sigue siendo grande entre la población.

Palabras clave: uso completo, reutilización de alimentos, desperdicio.

\section{Introdução}

O direito à alimentação adequada é um direito humano a todas as pessoas de ter acesso regular, permanente a alimentos seguros e saudáveis, em quantidade e qualidade adequadas e suficientes, correspondentes às tradições culturais do seu povo e que garanta uma vida livre do medo, digna e plena nas dimensões física e mental, individual e coletiva (Abrandh, 2010; Siqueira et al., 2014).

O aproveitamento integral dos alimentos é a utilização do alimento em sua totalidade e tem sido adotado como uma prática sustentável e ecologicamente correta, com uma maior utilização de recursos naturais, o que permite a redução de gastos com alimentação da família e estimula a diversificação dos hábitos alimentares, melhorando a qualidade nutricional (Santana \& Oliveira, 2005; Franzosi, Daneluz \& Baratto, 2018). Entretanto, a falta de informações sobre os princípios nutritivos e o aproveitamento dos alimentos gera o desperdício de toneladas de recursos alimentares (Nunes, 2009; Gomes \& Teixeira, 2017).

No Brasil, as perdas de alimentos começam no campo, por ocasião da colheita, e no preparo do produto para a comercialização, prosseguindo na rede de transporte, nas centrais de abastecimento e em outros atacadistas, e finalmente na rede varejista e por consumidores intermediários e finais (Vilela et al.,2003; Silva et al., 2017). O desperdício é um sério problema a ser resolvido na produção e distribuição de alimentos, principalmente nos países subdesenvolvidos ou em desenvolvimento (Carvalho et al., 2009). Nesta cadeia produtora, o consumidor também contribui para o desperdício, pois cerca de $60 \%$ dos alimentos adquiridos nas residências vão para o lixo (Santana \& Oliveira, 2005). Uma das contribuições para o 
desperdício de alimentos em domicilio acontece quando a dona de casa tende a comprar produtos hortícolas em excesso, favorecendo a perdas por deterioração. Igualmente despreza folhas, cascas e talos, devido a tabus alimentares ou desconhecimento sobre sua utilidade como alimento, além das sobras de refeições que também são desprezadas (Prim, 2003; Jesus \& Pires, 2018).

Apesar do Brasil ser considerado um país rico em diversidade e quantidade de alimentos produzidos, milhares de brasileiros passam fome, e aproximadamente $20 \%$ dos grãos e 30\% das hortaliças são desperdiçadas (Vilhena \& Silva, 2007). Uma alternativa que vem ganhando corpo desde o início de 1970 consiste no aproveitamento de partes desprezadas dos alimentos como matéria-prima para a produção de algumas receitas perfeitamente passíveis de serem incluídos na alimentação humana (Malucelli et al., 2009; Farias et al., 2016).

No Brasil, iniciou-se o uso do aproveitamento integral de alimentos no combate a fome e às carências nutricionais principalmente para os grupos mais vulneráveis aos desníveis sociais como gestantes e crianças. Com o passar do tempo e com as experiências adquiridas via da utilização integral, este trabalho estendeu-se por todo o país e deixou de ser indicado apenas para a população de baixa renda, mas para toda a população, pois além de ser economicamente importante, do ponto de vista nutricional representa benefícios para toda população (Nunes, 2009; Cardoso \& Vieira, 2019).

A falta do hábito de utilizar adequadamente os alimentos, aproveitando praticamente todas as suas partes é um dos motivos do desperdício (Vilhena \& Silva, 2007; Faria et al., 2016). Pouca atenção é destinada às partes vegetativas de algumas espécies de hortaliças, consideradas de alto valor nutritivo (Malucelli et al., 2009).

$\mathrm{O}$ aproveitamento integral de alimentos pode enriquecer a alimentação diária, com inúmeros benefícios, além de reduzir o desperdício e proporciona uma maior diversidade de nutrientes na dieta (Alves et al., 2007). O estudo da utilização integral de hortaliças, verduras e frutas no uso doméstico, considerando a importância econômica e nutricional do aproveitamento das partes usualmente desprezadas, bem como sua incorporação na elaboração de produtos industrializados, pode contribuir substancialmente para aumentar a disponibilidade de nutrientes, sendo fonte de proteínas, fibras, vitaminas e minerais (Perreira et al., 2003; StorckI et al., 2013).

Nesse sentido o presente estudo teve como objetivos conhecer as práticas de aproveitamento integral, o desperdício de alimentos e o destino dado as sobras alimentares pelos moradores de bairros da cidade de Diamantina/MG. 


\section{Métodos}

\subsection{Local e população de estudo}

O estudo foi realizado na cidade de Diamantina/MG, localizada na Mesorregião do Jequitinhonha, no período de agosto de 2011 a dezembro de 2012. Tratou-se de um estudo exploratório e descritivo, como apresenta Pereira et al. (2018), no qual os participantes eram moradores de 4 bairros atendidos por unidades de saúde da família da cidade de Diamantina, sendo a amostra por conveniência definida por um número de 50 pessoas por bairro.

A seleção dos participantes foi realizada por meio de sorteio aleatório simples tendo como base o cadastro das famílias pela Unidade de Saúde. O instrumento de coleta foi um formulário semi-estruturado constando de dois blocos, o primeiro relativo às características socioeconômicas e demográficas, e o segundo, relativo às práticas de preparo, conservação, consumo de alimentos e destino das sobras.

O formulário foi aplicado com indivíduos maiores de 18 anos e após a assinatura do Termo de Consentimento Livre e Esclarecido (TCLE). Os formulários foram aplicados por alunas dos cursos de Nutrição e Enfermagem devidamente treinadas para essa prática.

\subsection{Variáveis do estudo}

As características socioeconômicas foram identificadas pelas seguintes variáveis: grau de escolaridade, sexo, idade, recebimento de benefício/auxílio, condições de moradia e saneamento básico.

Em relação aos hábitos de consumo, as variáveis estudadas foram relativas: ao aproveitamento, de alimentos de forma integral; reaproveitamento de alimentos; alimentos mais comumente perdidos antes e após serem preparados, hábitos de compra como verificação de data de validade, responsável pela aquisição de gêneros alimentícios no domicilio, o que significa o uso integral de alimentos, destino das sobras e a preocupação de se evitar o desperdício de alimentos.

\subsection{Análises estatísticas}

A constituição do banco de dados foi realizada utilizando o programa Microsoft Office Excell $2007^{\circledR}$. A obtenção de medidas de frequência simples e relativa bem como medidas de 
tendência central como média e mediana foram obtidas utilizando o programa estatístico STATA $9.0^{\circledR} 2008$.

\subsection{Aspectos éticos}

O projeto foi submetido e aprovado pelo Comitê de Ética em Pesquisa da Universidade Federal dos Vales do Jequitinhonha e Mucuri e seu parecer consubstanciado recebido o número 064/2011.

\section{Resultados}

Dos 200 domicílios sorteados, 181 participaram do estudo, sendo que nos demais 19 (9.5\%) não foram localizados moradores ou os mesmo não aceitaram participar da pesquisa. Dos 181 domicílios, 84,5\% dos entrevistados eram do sexo feminino e 15,4\% do sexo masculino. A idade média foi de 49,9 anos $( \pm 19,8)$ com idade mínima de 18 anos e máxima de 88 anos. Em relação aos anos completos de estudos a média foi de 7,8 anos $( \pm 5,4)$.

Dos entrevistados apenas $15,4 \%$ residiam sós, sendo, portanto a única pessoa residente no domicílio. A média de pessoas por residência foi de 2,6 pessoas $( \pm 1,8)$. Quanto ao recebimento de algum benefício/auxílio, 64,6\% dos participantes disseram receber e 35,3\% não o recebiam. Dos 181 domicílios participantes todos possuíam casa de alvenaria, água encanada em domicílio, energia elétrica, coleta de lixo e esgotamento sanitário.

Quanto ao aproveitamento integral dos alimentos, a perda de alimentos em casa antes do consumo foi relatada por $41,4 \%$ dos participantes, sendo as verduras de folhas o tipo de alimento mais citado $(62,1 \%)$ como mostra a Tabela 1 .

Tabela 1 - Distribuição dos alimentos segundo o percentual de perdas antes do consumo, Diamantina, MG, 2012.

\begin{tabular}{lcc}
\hline Tipo de alimento & \multicolumn{3}{c}{ Frequência } \\
\cline { 3 - 3 } & $\mathbf{N}$ & $\mathbf{\%}$ \\
\hline Verdura de Folha & 23 & 62,1 \\
Legumes & 7 & 18,9 \\
Frutas & 7 & 18,9 \\
Total & 37 & 100 \\
\hline
\end{tabular}

Fonte: Autores. 
Research, Society and Development, v. 9, n. 7, e201973757, 2020

(CC BY 4.0) | ISSN 2525-3409 | DOI: http://dx.doi.org/10.33448/rsd-v9i7.3757

Dentre os alimentos que mais se perderam antes do preparo/consumo a alface foi a mais citada entre os participantes $(19,3 \%)$, seguida pelo tomate $(14,2 \%)$ e a couve $(8,4 \%)$ (Tabela 2).

Tabela 2 - Frequência dos alimentos perdidos antes do preparo/consumo, Diamantina, MG, 2012.

\begin{tabular}{|c|c|c|}
\hline \multirow[t]{2}{*}{ Alimentos } & \multicolumn{2}{|c|}{ Frequência } \\
\hline & $\mathbf{N}$ & $\%$ \\
\hline Alface & 23 & 19,33 \\
\hline Tomate & 17 & 14,29 \\
\hline Couve & 10 & 8,4 \\
\hline Batata & 8 & 6,72 \\
\hline Abobora & 8 & 6,72 \\
\hline Pimentão & 6 & 5,04 \\
\hline Cenoura & 6 & 5,04 \\
\hline Arroz & 4 & 3,36 \\
\hline Maça & 4 & 3,36 \\
\hline Feijão & 4 & 3,36 \\
\hline Quiabo & 3 & 2,52 \\
\hline Repolho & 3 & 2,52 \\
\hline Banana & 3 & 2,52 \\
\hline Laranja & 3 & 2,52 \\
\hline Abacaxi & 2 & 1,68 \\
\hline Jiló & 2 & 1,68 \\
\hline Chuchu & 2 & 1,68 \\
\hline Melancia & 2 & 1,68 \\
\hline Outros Alimentos & 3 & 2,52 \\
\hline Total & 114 & 100 \\
\hline
\end{tabular}

Fonte: Autores.

Em relação ao preparo de refeições $62,4 \%$ dos participantes relataram preparar refeições de forma que haja sobras. Os alimentos que mais frequentemente sobravam eram o $\operatorname{arroz}(47,2 \%)$ e as saladas $(18,1 \%)$ (Tabela 3$)$. 
Research, Society and Development, v. 9, n. 7, e201973757, 2020

(CC BY 4.0) | ISSN 2525-3409 | DOI: http://dx.doi.org/10.33448/rsd-v9i7.3757

Tabela 3 - Distribuição das preparações segundo a frequência de sobras, Diamantina, MG, 2012.

\begin{tabular}{lcc}
\hline Preparações & \multicolumn{2}{c}{ Frequência } \\
\cline { 2 - 3 } & N & \% \\
\hline Arroz & 26 & 47,2 \\
Saladas & 10 & 18,1 \\
Verduras Refogadas & 9 & 16,4 \\
Feijão & 7 & 12,7 \\
Macarrão & 2 & 3,6 \\
Carne & 1 & 1,8 \\
Total & 55 & 100 \\
\hline
\end{tabular}

Fonte: Autores.

Quando perguntados sobre a afirmação popular "antes sobrar do que faltar", a maioria $(91,1 \%)$ referiu concordar.

Sobre o destino das sobras de alimentos $71,2 \%$ dos participantes relataram fazer o reaproveitamento das sobras, $6 \%$ era doado para o consumo de outras pessoas, $14,9 \%$ usado para alimentação animal e 10,5\% descartado.

Dos participantes que responderam reaproveitar as sobras, $58 \%$ a utilizavam para uma nova preparação, já $42 \%$ disseram reaproveitar a comida, mas não em uma nova preparação.

Quando perguntados sobre a data de validade de alimentos, 58,6\% dos responderam que descartam o alimento com data de validade vencida e 41,4\% não descartavam o alimento mesmo com a data de validade vencida.

Ainda sobre data de validade do produto $77,9 \%$ dos participantes afirmaram ter o hábito de olhar data de validade.

O aproveitamento integral dos alimentos foi relatado por $47,2 \%$ dos participantes, sendo a abobrinha Itália o alimento mais citado com $36,3 \%$ seguida da couve com 18,1\% (Tabela 4). 
Tabela 4 - Distribuição dos alimentos segundo a frequência de seu uso integral, Diamantina, MG, 2012.

\begin{tabular}{lcc}
\hline Alimentos & \multicolumn{2}{c}{ Frequência } \\
\cline { 2 - 3 } & $\mathbf{N}$ & $\mathbf{\%}$ \\
\hline Abobrinha & 56 & 36,36 \\
Couve & 28 & 18,18 \\
Chuchu & 14 & 9,09 \\
Jiló & 12 & 7,79 \\
Tomate & 10 & 6,49 \\
Batata & 10 & 6,49 \\
Cenoura & 4 & 2,6 \\
Pepino & 4 & 2,6 \\
Taioba & 3 & 1,95 \\
Maça & 3 & 1,95 \\
Abacaxi & 2 & 1,3 \\
Mostarda & 2 & 1,3 \\
Berinjela & 1 & 0,65 \\
Laranja & 1 & 0,65 \\
Salsinha & 1 & 0,65 \\
Mamão Verde & 1 & 0,65 \\
Beterraba & 1 & 0,65 \\
Espinafre & 1 & 0,65 \\
Total & 154 & 100 \\
\hline Fonte: Aut & &
\end{tabular}

Fonte: Autores.

\section{Discussão}

Entende-se por perdas, a parte física da produção que não é destinada ao consumo, em razão de depreciação da qualidade dos produtos, devido à deterioração, causada por amassamentos, cortes, podridões e outros fatores. Os alimentos são desperdiçados, quando, embora em boas condições fisiológicas, não são totalmente aproveitados pelo consumidor a que se destinam sendo desviados do consumo para o lixo (Vilela et al., 2003; Neris et al., 2018).

No presente estudo quase metade dos entrevistados relatou ter perda de alimentos antes do consumo, sendo a verdura de folha a perda mais citada. Esse perfil foi também descrito em estudo realizado pela EMBRAPA (Soares, 2007) que mostrou a produção das principais hortaliças frescas (folha, flor, fruto, haste, raízes e rizoma) comercializadas no Brasil é de aproximadamente 16,0 milhões de toneladas, dessa produção há uma perda de 
$35 \%$ desses produtos, sendo assim, tem-se uma estimativa de 5,6 milhões de toneladas/ano de produto não consumido $-37 \mathrm{~kg} / \mathrm{hab} / \mathrm{ano}$.

Neste caso verifica-se que se descartaram mais hortaliças do que se consome no Brasil. O percentual de perdas de hortigranjeiros "in natura" no Brasil registrado no estudo foi de $45 \%$ para a alface, $50 \%$ couve-flor, $40 \%$ pimentão e tomate, $35 \%$ repolho (Soares, 2007).

No estudo de Vilela et al., (2003) foi observado que no âmbito dos domicílios, a taxa de perdas é de $20 \%$ no consumo final dos produtos hortícolas. O desperdício em âmbito domiciliar pode ser gerado, pela guarda do produto fora de refrigeração e de forma inadequada, pela falta de planejamento das compras e o não aproveitamento das partes dos vegetais não consideradas comercializáveis, como folhas e talos.

Ao deixar de planejar as compras de produtos vegetais, o consumidor ou compra uma quantidade além de suas necessidades, ou faz suas compras para um período muito longo, em ambos os casos haverá deterioração ou prejuízo na aparência do produto, ocasionando perdas (Prim, 2003; Lana e Moita, 2019).

De acordo com o Mesa Brasil (2008) dos alimentos comprados pelas famílias brasileiras 30 a $40 \%$ destes vão parar no lixo, sendo assim o lixo domiciliar é composto predominantemente por comida (cascas, folhas, talos, sementes, bagaços entre outros) sendo que a cada ano 26,3 milhões de toneladas de restos de alimento são desperdiçados, esse índice não chega a $10 \%$ em outros países como os Estados Unidos. O Brasil é um dos maiores produtores de alimentos do mundo.

Um dos fatores que motivam o desperdício de alimentos durante o preparo é cultural, a população de modo geral não tem o hábito de aproveitar sobras e as partes menos valorizadas dos alimentos, como cascas, folhas ou talos. Além disso, permanece na cultura popular que se tem que adquirir mais alimentos do que irá consumir. Vê-se, então, que a melhoria das condições nutricionais da população brasileira passa também pela conscientização quanto às perdas e ao desperdício de alimentos (SESI Cozinha Brasil, 2008).

Em Unidade de Alimentação e Nutrição (UAN), o desperdício é proveniente da sobra de alimentos, ou seja, alimentos preparados e não distribuídos, ou restos, alimentos distribuídos e não consumidos (Vargas e Hautrive, 2011; Zanini eta al., 2017).

Quantidades significativas de restos de alimentos justificam a importância do gerenciamento no controle de desperdício. Uma avaliação diária de restos é uma medida a ser utilizada no controle do desperdício (Moura et al., 2009). 
Também considerando uma UAN, o desperdício é sinônimo de falta de qualidade e, deve ser evitado por meio de um planejamento adequado, a fim de que não existam excessos de produção e consequentes sobras (Ricarte et al., 2008; Zanini eta al., 2017). Outro aspecto a ser considerado ao se tratar de desperdício de alimentos, refere-se ao treinamento dos colaboradores, este proporciona informações aos empregados sobre as técnicas dietéticas, visando diminuir e /ou evitar o desperdício de gêneros alimentícios e resultados danosos ao valor nutritivo das refeições (Vargas e Hautrive, 2011).

Os resultados do presente estudo mostram que a maioria dos participantes faziam refeições de forma que houvesse sobras e, dentre as que mais sobravam eram as saladas e o arroz. As sobras limpas são alimentos produzidos e não distribuídos.

Em uma Unidade de Alimentação e Nutrição (UAN) a quantidade de sobras limpas deve estar relacionada ao número de refeições servidas e à margem de segurança, definida na fase de planejamento.

Os registros das quantidades de sobras limpas diárias são fundamentais, pois servem como subsídios para implantar medidas de racionalização, redução de desperdícios e otimização da produtividade (Augustine et al., 2008 ).

Segundo Carmo e Lima (2011) em estudo de avaliação de sobras limpas em uma UAN na cidade de Campo Grande/MS, as sobras limpas no final de 20 dias de avaliação foram de $77,8 \mathrm{~kg}$ para o arroz, 94,3kg para o feijão, 65,7kg para carnes, guarnição 53,4kg e hortaliças $66,5 \mathrm{~kg}$.

Ricarte et al., (2008) em seu estudo mostraram que as sobras limpas de saladas em média por dia foi de $5 \mathrm{~kg}$, o que representa aproximadamente $7 \%$ da quantidade produzida diariamente.

Apesar dos estudos compararem ambientes diferentes, observa-se que o registro de sobras limpas em ambos são parecidos, ou seja, tanto em domicilio como numa unidade de UAN as refeições que tiveram sobras de mais frequentemente foram saladas e o arroz. Com isso pode-se observar que os hábitos alimentares da população tendem a ser iguais quando comparados em âmbito domiciliar ou em restaurantes.

Entre a população aqui estudada, o reaproveitamento de alimentos é uma prática comum, sendo que a maioria relatou fazer uso desta prática, seja para produção de uma nova preparação ou não.

O reaproveitamento de sobras limpas deve ter um controle de tempo e temperatura para que os microrganismos não se multipliquem excessivamente até o consumo. Considerase que o reaquecimento inadequado de um alimento servido um ou mais dias após a sua 
preparação, ou daquele que resta da refeição anterior, é muitas vezes, identificado como veiculo de toxinfecções. Se as bactérias tiverem sobrevivido á cocção inicial, ou se houver uma contaminação após o aquecimento ou resfriamento inadequado, as toxinas devem ser eliminadas durante o seu reaquecimento (Carmo \& Lima, 2011).

Segundo Araujo et al., (2007) a forma mais comum do desperdício domiciliar é a falta do conhecimento de se reaproveitar e utilizar o alimento integralmente, para elaboração de pratos nutritivos que sejam atrativos visualmente, e saborosos com o que seria desperdiçado.

De acordo com o presente estudo cerca de metade dos entrevistados disseram aproveitar integralmente os alimentos. Esse índice também pode ser observado no estudo de Gonçalves e Torres (2010) sobre o aproveitamento de alimentos entre acadêmicos, onde $61 \%$ dos alunos de vários cursos responderam fazer o preparo de alguma refeição aproveitando as cascas, talos e sementes de alimentos, já entre os alunos do curso de Nutrição esse índice subiu para $85 \%$.

Apesar das possíveis diferenças em termos de conhecimentos, observa que a prática do aproveitamento integral ainda é pouco frequente na população em geral quando comparada aos grupos de estudantes. Essa diferença mostra que ainda e preciso mais estudos para um maior conhecimento e prática dessa alimentação.

Segundo Oliveira et al., (2011) essa prática é sem dúvida uma importante atitude de melhoria da saúde das pessoas, gerando uma diminuição do lixo e consequentemente um aumento da qualidade de vida das famílias.

No estudo de Viera et al., (2010), sobre a análise sensorial de sucos elaborados com aproveitamento integral de alimentos observaram que os sucos elaborados foram uma forma encontrada de oferecer uma alimentação mais saudável, aproveitando nutrientes contidos em folhas, talos e cascas de frutas, nos quais têm-se por hábito descartar.

Dentre os sucos elaborados verificou-se que o suco da casca de maçã apresentou índice de aceitabilidade $35,82 \%$, o suco de couve com limão 57,49\%, e o refrigerante caseiro (cenoura e laranja) obteve a maior aceitação com $81,65 \%$.

A abobrinha Itália e a couve foram os alimentos mais citados pelos participantes quando perguntados sobre o uso integral. Em pesquisa realizada pelo Instituto Akatu (2003) com mais de 1200 pessoas revelou que 60\% dos participantes já ouviram falar em aproveitamento integral de alimentos, porém apenas 8,3\% disseram que aproveitam os talos, sementes, cascas e folhas dos alimentos.

Estudo de Gondim et al., (2007) sobre composição centesimal de minerais em casca de frutas observaram que vitaminas, minerais e fibras podem ser encontrados amplamente nos 
talos e cascas de alimentos, partes que comumente são descartadas. Muitas vezes, o teor de alguns nutrientes na casca e nos talos é ainda maior do que na polpa do respectivo alimento como é o caso das fibras, potássio, cálcio e magnésio.

O incentivo ao aproveitamento integral pode trazer contribuições para diminuir os gastos com alimentação e melhorar a qualidade nutricional do cardápio, além de reduzir o desperdício de alimentos.

A alimentação saudável pode ser acessível física e economicamente à sociedade como um todo e tem impacto importante sobre os principais fatores de risco à saúde, muitos deles comuns a várias doenças, além de ser saborosa e respeitar a cultura alimentar da pessoa, de sua família e do seu grupo social (Abrandh, 2010).

Os alimentos devem ser seguros para o consumo, ou seja, não devem apresentar contaminantes de natureza biológica, física ou química ou outros perigos que comprometam a saúde do indivíduo ou da população. Nesse sentido, com o objetivo de redução dos riscos à saúde, medidas preventivas e de controle, incluindo as boas práticas de higiene, devem ser adotadas em toda a cadeia de alimentos, desde a sua origem até o preparo para o consumo em domicílio, em restaurante e em outros locais que comercializam alimentos (Abrandh, 2010).

Nossos achados mostram que mais da metade dos participantes disseram que o uso integral dos alimentos é uma forma de tornar as refeições mais saudáveis. No estudo de Nunes (2009) sobre aproveitamento integral dos alimentos 67,9\% dos alunos entrevistados informou que já tinha ouvido falar sobre o aproveitamento integral dos alimentos e ressaltaram que o seu significado é aproveitar o alimento em sua totalidade levando a uma refeição mais saudável. Já 57,1\% dos participantes responderam que é uma forma de evitar o desperdício, pois aproveita o máximo dos alimentos.

Portanto, assim como nesse estudo, outros autores identificaram que o conhecimento do valor do uso integral dos alimentos tanto para melhorar a qualidade da alimentação como para diminuir o desperdício foi documentada entre os entrevistados.

Apesar do conhecimento, a prática ainda não é majoritária na população em geral, o que demonstra um espaço em aberto para ações educativas relativas á essa prática. Nesse sentido, concomitante a presente pesquisa, realizou-se atividades de extensão, promovendo oficinas de uso integral e reaproveitamento seguro dos alimentos, buscando difundir essa prática entre a população local. 


\section{Conclusão}

O presente estudo trouxe uma discussão sobre a importância da prática do aproveitamento integral de alimento integral, evitando-se assim o desperdício de alimentos, quando a sobras destes são destinados a outros fins alimentares.

Neste contexto, reforça-se a importância dessa discussão interdisciplinar que perpassa pelas áreas de ciências da nutrição, bem como da agroecologia e ciências sociais, visto que o desperdício e o reaproveitamento dos alimentos possuem uma conexão com diversas áreas do conhecimento. Entretanto, tem sido discutido pouco sobre o aproveitamento integral dos alimentos, e esta é uma prática pouco difundida em nosso meio, sendo frequente o descarte de partes de alimentos que podem ser usados na alimentação.

Com relação aos achados do presente estudo, conclui-se que a perda de alimentos em casa antes do preparo/consumo foi observada em uma parcela considerável dos participantes, e a minoria pratica o aproveitamento integral dos alimentos.

Assim, reforça-se a importância dessa discussão sobre o aproveitamento integral dos alimentos, visto que isso poderia contribuir de forma importante com a oferta de nutrientes bem como com a redução do desperdício no domicílio.

Neste contexto, reforça-se a necessidade do desenvolvimento de novos estudos para ampliar o conhecimento acerca do uso integral e reaproveitamento seguro dos alimentos bem como ações para a difusão dos benefícios relacionados a essa prática interdisciplinar.

\section{Agradecimentos}

À Fundação de Amparo à Pesquisa do Estado de Minas Gerais (FAPEMIG) pelo recurso financeiro através do edital 07/2011, processo APQ-02881-11.

À Pro-Reitoria de Extensão e Cultura (PROEXC) da Universidade Federal dos Vales do Jequitinhonha e Mucuri pelo recurso financeiro.

\section{Referências}

Abrandh. (2010). Direito Humano á Alimentação Adequada no Contexto da Segurança Alimentar e Nutricional. Ação Nutrição e Direitos Humanos (Abrandh)Brasília/DF: 2010. 
Alves, JA, Novello, D, Ost, PR. \& Quintiliano, DA. (2007). Aceitação de torta de farinha integral feita com reaproveitamento de alimentos por crianças pré-escolares em uma creche municipal do município de Guarapuava-PR. Alim. Nutr. 18(2): 161-166.

Araujo, MEM, Rocha, AMP \& Wesz, RS. (2007). Projeto alimentação inteligente = desperdício zero. Instituto Federal de Educação, Ciência e Tecnologia Farroupilha, Campus São Vicente do Sul, 2007.

Augustini, VCM, Kishimoto, P, Tescaro, TC \& Almeida, FQA. (2008). Avaliação do índice de resto-ingesta e sobras em uma unidade de alimentação e nutrição (UAN) de uma empresa metalúrgica na cidade de Piracicaba/SP. Rev. Simbio-Logias. 1(1): 99-110.

Cardoso, AMA. \& Vieira, TA. (2019). Práticas de redução do desperdício de alimentos: o caso de um projeto social em Santarém, Pará. Multitemas. 24(58).

Carmo, SO, Lima, TP. (2011). Avaliação do índice de sobras limpas em uma unidade de alimentação e nutrição (UAN) institucional na cidade de Campo Grande-MS. Ensaios $e$ Ciências: Ciências Agrárias, Biológicas e da Saúde. 15(6): 9-20.

Carvalho, LMS, Silva, LO, Dias, LP \& Cronemberger, MGO. (2009). Aproveitamento alternativo da batata inglesa para elaboração de bolo frito. In: $4^{\circ}$ Congresso de Pesquisa e Inovação da Rede Norte Nordeste de Educação Tecnológica (CONNEPI), 2009, Belém/PA.

Farias, PKS, Souza, SDO, Santana, IMO, Prates, RP, Gusmão, ACM \& Soares, PDF. (2016). Desenvolvimento e análise sensorial de diferentes tipos de hambúrgueres funcionais utilizando o reaproveitamento de alimentos. Cad. Ciênc. Agra. 8(3): 07-14.

Franzosi, D.; Daneluz. H. C.; Baratto, I. (2018). Níveis de desperdício de partes não convencionais de produtos utilizados diariamente em um restaurante no sudoeste do Paraná. Revista Brasileira de Obesidade, Nutrição e Emagrecimento. 12(69): 66-75.

Gomes, MEM, Teixeira, C. (2017). Aproveitamento integral dos alimentos: qualidade nutricional e consciência ambiental no ambiente escolar. Ensino, Saúde e Ambiente. 10(1), 203-217. 
Gonçalves, FL \& Torres, AAL (2010). Aproveitamento integral dos alimentos entre acadêmicos de uma faculdade particular do DF. [Tese]. Brasília: Curso de Educação e Promoção da Saúde, Universidade Federal de Brasília, 2010.

Gondim, JAM, Moura, MFV, Dantas, AS; Medeiros, RLS, Santos, KM. (2005). Composição centesimal e de minerais em cascas de frutas. Ciênc. Tecnol. Aliment. 25(4): 825-827.

Instituto Akatu. (2003). Caderno temático. A nutrição e o consumo consciente. Pinheiros (São Paulo). 2003.

Jesus, C., Pires, I. (2018). "Fechar o ciclo". A contribuição da economia circular para o combate ao desperdício alimentar. Revista Ecologias Humanas. 4(4): 7-20.

Lana, MM \& Moita, AW. (2019). Visual quality and waste of fresh vegetables and herbs in a typical retail market in Brazil. Horticultura Brasileira. 37(2).

Malucelli, M, Novello, D, Ando, N, Almeida, JM \& Freitas, AR. (2009). Avaliação e composição nutricional de nhoque tradicional e enriquecido com farinha de resíduo de brócolis (Brassica oleracea var. Italica). Alim. Nutr. 20(4): 553-560.

Mesa Brasil Sesc. (2008). Programa Sesi cozinha Brasil, Brasília.

Moura, PN, Honaiser, A \& Bolognini, MCM. (2009). Avaliação do índice de resto ingestão e sobras em unidade de alimentação e nutrição (UAN) do colégio agrícola de Guarapuava (PR). Revista Salus-Guarapuava. 3(1): 15-22.

Navolar, TS, Tesser, CD, Azevedo, E. (2012). Contribuições para a construção da nutrição integrada. Comunicação Saúde Educação. 16(41): 515-527.

Neris, TS, Silva, SS, Loss, RA, Carvalho, JWP, Guedes, SF. (2018). Avaliação físico-química da casca da banana (Musa spp.) in natura e desidratada em diferentes estádios de maturação. Ciência e Sustentabilidade. 4(1): 5-21. 
Nunes, JT. (2009). Aproveitamento integral dos alimentos: qualidade nutricional e aceitabilidade das preparações. [Monografia]. Brasília: Centro de Excelência em Turismo CET, Universidade Federal de Brasília, 2009.

Oliveira, CCA, Silva, JM, Reis, TC, Nunes, JEA \& Lima, DES. (2011). Aproveitamento integral dos alimentos: contribuições para melhoria da qualidade de vida e meio ambiente de um grupo de mulheres da cidade do Recife - PE. 2011. Acesso em: 02 maio 2020. Disponível em: http://www.xxcbed.ufc.br/arqs/public/t_07.pdf.

Pereira, GIS, Pereira, RGFA, Barcelos, MFP \& Morais, AR. (2003). Avaliação química da folha de cenoura visando ao seu aproveitamento na alimentação humana. Ciênc. Agrotec. 27(4): 852-857.

Pereira, AS, Shitsuka, DM, Parreira, FJ \& Shitsuka, R. (2018). Metodologia da pesquisa científica. [e-book]. Santa Maria. Ed. UAB/NTE/UFSM. Acesso em: 2 maio 2020. Disponível em: https://repositorio.ufsm.br/bitstream/handle/1/15824/Lic_Computacao_MetodologiaPesquisa-Cientifica.pdf?sequence $=1$.

Prim, MBS. (2003). Analise do desperdício de partes vegetais consumíveis. [Dissertação]. Florianópolis: Universidade Federal de Santa Catarina, 2003.

Ricarte, MPR, Fé, MABM \& Santos, IHVS \& Lopes, AKM. (2008). Avaliação do desperdício de alimentos em uma unidade de alimentação e nutrição institucional em Fortaleza - CE. Saber Científico.1(1): 158-175.

Santana, AF \& Oliveira, LF. (2005). Aproveitamento da casca de melancia (Curubita citrullus, Shrad) na produção artesanal de doces alternativos. Alim. Nutr. 16(4): 363-368.

Siqueira, RL, Cotta, RMM, Ribeiro, RCL, Sperandio, N \& Priore, SE. (2014). Análise da incorporação da perspectiva do Direito Humano à Alimentação Adequada no desenho institucional do Programa Nacional de Alimentação Escolar. Ciência \& Saúde Coletiva. 19(1): 301-310. 
Silva, RA, Nazareno, LSQ, Sousa, FA \& Rufino, MS. (2017). Análise do desperdício de hortifrútis proveniente da agricultura familiar no município de Capistrano - CE. Iniciação Científica UNICESUMAR. 19(1): 25-33.

Soares, AG.(2007). Desperdício de alimentos no Brasil - um desafio político e social a ser vencido. Embrapa [pesquisa]. Acesso em: 02 maio 2020. Disponível em: http://atividaderural.com.br/artigos/508fc56454d19.pdf.

StorckI, CR, Nunes, GL, Oliveira, BB \& Basso, C. (2013). Folhas, talos, cascas e sementes de vegetais: composição nutricional, aproveitamento na alimentação e análise sensorial de preparações. Ciência Rural. 43(3): 537-543.

Vargas, A \& Hautrive, TP. (2011). Analise e controle de sobras de alimentos em uma empresa fornecedora de refeições transportadas no município de Chapecó - SC. Revista de Tecnologia Agroindustrial. 5(2): 531-541.

Vieira, VB, Tambara, TT, Buzatti, NB, Barbosa, ALR, Lopes, MR, Miron, VR \& Saccol, ALF. (2010). Analise sensorial de sucos elaborados com aproveitamento integral dos alimentos. In: $3^{\circ}$ Jornada Interdisciplinar da Saúde; Santa Maria/RS, 2010.

Vilela, NJ, Lana, MM \& Makishima, N. (2003). O peso da perda de alimentos para a sociedade: o caso das hortaliças. Horticultura Brasileira. 21(2): 141-143.

Vilhena, MO \& Silva, MC. (2007). Aproveitamento integral de alimentos orgânicos: arte culinária verde. In: $2^{\circ}$ Jornada Nacional da Produção Cientifica em Educação Profissional e Tecnológica, 2007, São Luís/MA.

Zanini, RR, Spohr, C, Peripolli, A, Furtado, JH, Noronha, MO \& Dapper, SN. (2017). Avaliação do desperdício alimentar produzido por comensais em restaurante universitário no Sul do Brasil por meio de gráficos de controle. Latin American Journal of Business Management. 8(2), 118-133. 
Research, Society and Development, v. 9, n. 7, e201973757, 2020

(CC BY 4.0) | ISSN 2525-3409 | DOI: http://dx.doi.org/10.33448/rsd-v9i7.3757

Porcentagem de contribuição de cada autor no manuscrito

Tássia Andrade Ferreira - 20\%

Renata Cristina Rodrigues - 20\%

Ronilson Ferreira Freitas - 20\%

Ana Catarina Perez Dias - 20\%

Angelina do Carmo Lessa - 20\% 